\title{
Delayed Administration of Nafamostat Mesylate Inhibits Thrombin-Mediated Blood-Spinal Cord Barrier Breakdown During Acute Spinal Cord Injury in Rats
}

\section{Chenxi Zhao}

Tianjin Medical University General Hospital

\section{Tiangang Zhou}

Tianjin Medical University General Hospital

\section{Xiaoqing Zhao}

Shandong University Cheeloo College of Medicine

\section{Yilin Pang}

Tianjin Medical University General Hospital

\section{Wenxiang Li}

Shandong University Cheeloo College of Medicine

\section{Baoyou Fan}

Tianjin Medical University General Hospital

\section{Ming Li}

Tianjin Medical University General Hospital

\section{Xinjie Liu}

Tianjin Medical University General Hospital

\section{Lei Ma}

Tianjin Medical University General Hospital

Jiawei Zhang

Tianjin Medical University General Hospital

\section{Chao Sun}

Tianjin Medical University General Hospital

\section{Wenyuan Shen}

Tianjin Medical University General Hospital

\section{Xiaohong Kong}

Shandong University School of Medicine: Shandong University Cheeloo College of Medicine

Xue Yao ( $\nabla$ xueyao@tmu.edu.cn )

tianjin medical university https://orcid.org/0000-0003-4904-7697

\section{Shiqing Feng}

Tianjin Medical University General Hospital 


\section{Research}

Keywords: Nafamostat Mesylate, Thrombin, Administration time window, Spinal cord injury, Blood spinalcord barrier, Neuroinflammation

Posted Date: November 23rd, 2021

DOI: https://doi.org/10.21203/rs.3.rs-1076149/v1

License: (c) (1) This work is licensed under a Creative Commons Attribution 4.0 International License. Read Full License 


\section{Abstract}

Background: Nafamostat mesylate (NM), an FDA-approved serine protease inhibitor, exerts antineuroinflammation and neuroprotective effect on rat spinal cord injury (SCl). However, the time window for $\mathrm{NM}$ administration after $\mathrm{SCl}$ as well as its underlying mechanism remains unclear.

Methods: A series of different first administration time points of NM was tested on rat contusive SCI model. The optimal time window of NM was screened by evaluating hindlimb locomotion and electrophysiology. We performed western blot and immunofluorescence to evaluate the drug target thrombin as well as its downstream Protease activated receptor 1 (PAR-1), and matrix metalloproteinase9 (MMP9). Enzyme activity assay was used to test thrombin activity. The permeability of blood-spinal cord barrier (BSCB) was assessed by Evans Blue leakage. The infiltration of peripheral inflammatory cell was observed by immunofluorescence.

Results: The optimal administration time window of NM was 2-12 h. The thrombin specific inhibitor, Argatroban, had similar pattern. The temporal expression pattern of thrombin peaked at 12 hours and returned to normal level at 7 days post SCI. PAR-1, the thrombin receptor, was observed a significant upregulation after SCl. MMP9, downstream of PAR-1, was also increased along with thrombin and PAR1. The most significant increase of thrombin expression was detected in vascular endothelial cells (ECs). NM significantly downregulated the thrombin and MMP9 expression as well as thrombin activity in the spinal cord, especially in ECs. NM administration at 2-12 h after SCl could inhibit the leakage of Evans blue in the epicenter and upregulate tight junction proteins (TJPs) expression. $8 \mathrm{~h}$ administration of NM effectively inhibited the infiltration of peripheral macrophages in the acute SCl.

Conclusions: Our study provided preclinical data of NM administration time window in SCI model, which is clinically relevant in the acute $\mathrm{SCI}$. We elucidated the protective mechanism of NM through BSCB protection and anti-neuroinflammation via thrombin intervention.

\section{Background}

Traumatic spinal cord injury ( $\mathrm{SCl}$ ) is a severe central nervous system (CNS) disease with no effective treatment currently $[1,2]$. At present, the most extensive clinical medication for $\mathrm{SCl}$ is methylprednisolone (MP), which has a degree of curative effect on inhibiting the acute inflammatory cascade[3]. However, its narrow administration time window ( $<8 \mathrm{hpi})$ and remarkable side effect limits its clinical application. Although a few compounds presented neuroprotective effect in animal SCI model[4], the clinical transformation is often hindered by their safety, impractical administration time window as well as unclear mechanism.

Since Nafamostat mesylate (NM), a synthetic spectrum serine protease inhibitor [5], has been widely used in patients with pancreatitis and disseminated intravascular coagulopathy, its administration safety was sufficiently guaranteed. In recent years, NM has been proved the neuroprotective and anti-inflammation effects on CNS diseases[6, 7]. NM can also improve neurological outcome and axonal regeneration after 
stroke and protect against acute cerebral ischemia via blood-brain barrier (BBB) protection[8]. In our previous study, NM inhibited neuroinflammation during acute phase and promote the locomotor function recovery post SCI [9]. However, its administration time window and repair mechanism in SCl remains elusive.

Thrombin, a widely studied serine protease targeted by NM, is a bioactive enzyme involved in multiple functions and restricted from the CNS under physiological conditions[10]. Recent research has proved its crucial role in traumatic brain injury (TBI), SCl, neurodegenerative diseases (Alzheimer's and Parkinson's diseases) and ischemic stroke[11, 12]. Further, thrombin can promote the astrocyte activation-induced glial scar hyperplasia, neuronal apoptosis and neuropathic pain through protease activated receptor 1 (PAR1)[13-15]. Meanwhile, Machida, T., et al reported that thrombin produces MMP9 by activating the downstream PAR1 pathway[16]. MMP9 (matrix metalloproteinase 9) participated in the degradation of extravascular matrix and tight junction proteins (TJPs), including Claudin-5 (CLD5), Occludin and zonulaoccludens-1 (ZO-1) [17], and played an unignorable effect on the BBB dysfunction. However, the temporal and spatial distribution of thrombin after $\mathrm{SCl}$ remains unclear, restricting the research and development of targeted drugs.

As a highly selective permeable vascular endothelial structure, blood-spinal cord barrier (BSCB) provides strong support for the homeostasis of the spinal cord microenvironment $[17,18]$. The impairment of BSCB aggravates the pathological process of $\mathrm{SCl}$, allowing various molecules and blood cells to enter the spinal cord [19]. In addition to the leakage of blood-derived leucocyte and monocytes infiltration[20], various types of proteases, such as thrombin, plasmin and kallikrein crossed the impaired BSCB resulting in a perpetual neurological deficiency in acute $S C I[9][14,21]$. Hence, early drug administration targeting the BSCB protection could be beneficial to inhibit the neuroinflammatory cascade and improve long-term neurological outcomes.

In this paper, we proved the optimal administration time window of $\mathrm{NM}$ in $\mathrm{SCl}$ repair, and demonstrated that NM promoted the BSCB protection and improved neurological function of rats with SCI. The beneficial effect of NM may partly be mediated by inhibiting thrombin and its downstream signaling pathway.

\section{Methods}

\section{Animals}

Female Wistar rats (8 weeks old, 200-220 g) were purchased from Vital River Laboratory Animal Technology Co., Ltd (Beijing, China). Animals were kept in an environment with a 12/12-h light-dark cycle (light on between 7:00-19:00) with a temperature of $20-25{ }^{\circ} \mathrm{C}$ and humidity of $40 \%-60 \%$. Standard laboratory food and tap water were given ad libitum. All experimental procedures involving rats were approved by the Ethics Committee of Tianjin Medical University (Tianjin, China, IRM-DWLL-2020080). The animal experimental protocols were performed according to the ARRIVE guidelines. 


\section{Spinal cord injury}

All surgeries were conducted under aseptic condition. After a deep anesthetization with $4 \%$ inhaled isoflurane (R510-22, RWD, Shenzhen, China), the skin overlying the dorsal side of the spine was shaved and disinfected before surgery. A $1 \mathrm{~cm}$ midline longitudinal incision was made centered on T10 through the skin and muscle covering T10 vertebrae. The spinal cord was exposed by a T10 vertebrae dorsal

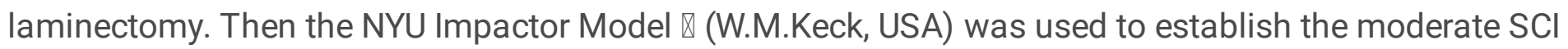
contusion model by a $10 \mathrm{~g}$ rod with a height of $25 \mathrm{~mm}$. The muscles, fascia, and skin were sutured layer by layer. Animals in Sham group were only performed with T10 laminectomy. Bladder care of animals was given twice daily until the end point of each experiment. Antibiotics (Gentamycin sulfate, Solarbio, G8170, Beijing, China) was administrated twice daily until 3 days post injury.

\section{Drug administration}

Nafamostat mesylate (S1386) and argatroban (MCl-9038), a thrombin-specific inhibitor, were purchased from Selleck (MA, USA). To explore the administration time window for NM and argatroban, we used different first administration times (FAT), including 0 h, 2 h, 8 h, 12 h, 24 h, 48 h, $5 \mathrm{~d}$ after SCl. Rats were randomly divided into groups of different FATs. NM $(1 \mathrm{mg} / \mathrm{mL}$, dissolved in $0.9 \%$ saline, $10 \mathrm{mg} / \mathrm{kg} / \mathrm{d})[9]$ and argatroban $(0.15 \mathrm{mg} / \mathrm{ml}$, dissolved in $0.9 \%$ saline, $1.5 \mathrm{mg} / \mathrm{kg} / \mathrm{d})$ [22] was administered by intraperitoneal (i.p.) injection at different FATs and then twice a day thereafter until 3 days post injury (dpi) or the endpoints of experiments. The rats in the Sham and SCl groups were injected with the same volume of $0.9 \%$ saline.

\section{Assessment of Basso, Beattie, and Bresnahan (BBB) locomotor score}

The Basso, Beattie, and Bresnahan (BBB) locomotor scores were used to evaluate the hind limb motor function [23]. BBB was performed before and 1 day and weekly post injury. The BBB test score with 0 represented no observable movement and 21 represented normal movement. Before score, rats were allowed to roam freely in an open field for 5 mins. Each rat was observed at least 2 mins and scored independently by at least 3 well-trained investigators. All the investigators were double-blinded.

\section{Catwalk-assisted gait analysis}

The locomotion recovery of rats in Sham, $\mathrm{SCl}$, and nafamostat, argatroban groups were also tested using the Noldus CatWalk XT system (version 10.6, Noldus, Netherlands) following the manufacturer's instructions (CatWalk XT 10.6 Reference Manual) and previous description[24]. Briefly, rats were trained to cross the walkway in the week before testing every day. Gait analysis was performed 5 weeks after SCl in a dark and quiet environment. Parameters including regularity index (RI), step sequence parameters, paw statistics, general parameters (mean speed and cadence), and bases of support were analyzed with CatWalk software version 10.6 by an observer blinded to the treatment.

\section{Electrophysiological tests}


Electrophysiological tests were assessed to indicate the nerve conduction function in rats 5 weeks post injury as previously described[25]. Sensory evoked potential (SEP) and motor evoked potential (MEP) of rats were processed by electrophysiological devices (YRKJ-G2008; Zhuhai Yiruikeji Co, Ltd, Guangdong, China). The recovery of sensory and motor nerve function in rats was evaluated according to the detection results. After the rats were deeply anesthetized and skin prepared, a $5.1 \mathrm{~Hz}$ square wave of 0.1 ms was generated by a constant stimulator $(2 \mathrm{~mA})$ in duration to stimulate the median nerve along the hindlimb for SEP and a single stimulator of $5 \mathrm{~mA}$ was used to stimulate the motor area of the cerebral cortex for MEP. For SEP, a total of 50 responses of each rat was averaged.

\section{Detection of coagulation function}

The effect of NM intervention on peripheral blood coagulation function of rats at different time points after injury was evaluated to determine the medication safety. Three rats were randomly selected from each group of rats administered at different time points, and $1 \mathrm{~mL}$ peripheral blood was collected from the endocanthal vein $2 \mathrm{~h}$ after the intervention. After the blood samples were centrifuged $(3000 \mathrm{rpm}, 10$ mins), an automatic coagulation analyzer (RAC030, Kolda, Wuhan, China) was used to detect the prothrombin time, prothrombin activity and international normalized ratio.

\section{Permeability of blood-spinal cord barrier}

The permeability of BSCB was investigated with Evans Blue (EB) according to the previous report[26]. Briefly, EB dye (E8010, $10 \mathrm{mg} / \mathrm{mL}$ diluted in saline, $1 \mathrm{~mL} / \mathrm{kg}$, Solarbio, Beijing, China) was administered through the tail vein and allowed to circulate for $3 \mathrm{~h}$. The spinal cord was resected out after deep anesthesia. The spinal cord was photographed from topical and coronal views to show EB leakage. The injured epicenter of spinal cord was sliced into $8 \mu \mathrm{m}$ sections under light protection. EB leakage was detected using a Leica fluorescence microscopy (Leica DMi8, Germany) under the excitation of $550 \mathrm{~nm}$ wave-length green light. The images were stitched together by Adobe Photoshop CC 2019. The total fluorescence intensity was measured by ImageJ (Version 1.46r; National Institutes of Health, Bethesda, $M D)$.

\section{Thrombin activity assay}

Thrombin activity was measured as previously described[27]. In brief, after perfused with $4{ }^{\circ} \mathrm{C}$ PBS, the injured spinal cord epicenter was removed and cut into $0.5 \mathrm{~cm}(20-30 \mathrm{mg})$. Epicenters were homogenized by assay buffer without the substrate ( $50 \mathrm{mM}$ Tris. $\mathrm{HCl}, \mathrm{pH} 5.8,150 \mathrm{mM} \mathrm{NaCl}, 1 \mathrm{mM} \mathrm{CaCl} 2,0.1 \% \mathrm{BSA})$. The homogenization was centrifuged at $12000 \mathrm{~g}$ for 13 mins at RT. Each $50 \mu \mathrm{L}$ sample was mixed with $150 \mu \mathrm{L}$ assay buffer mixed with the substrate (protease inhibitor prolyl endopeptidase inhibitor $\mathbb{Q}(20$ $\mathrm{mM})$, Benzoyl-Phe-Val-Arg-AMC· $\mathrm{HCl}(13 \mathrm{mM}))$. The mixture was added to a 96-well black microplate. The kinetic fluorescence was determined for 45 mins at $27^{\circ} \mathrm{C}$ by a fluorescence detection system (SYNERGY, BioTek; excitation 360/40 nm, emission 450/40 nm). Thrombin substrate Benzoyl-Phe-Val-Arg-AMC.HCl (B7632) and Prolyl Endopeptidase Inhibitor II (537011) were purchased from Merck (Germany). 


\section{Immunofluorescence staining}

The rats were perfused with $4^{\circ} \mathrm{C}$ PBS, followed by $4 \%$ paraformaldehyde. $0.5 \mathrm{~cm}$ spinal cord segment was resected from the level of epicenter and postfixed in $4 \%$ paraformaldehyde solution overnight. The samples were dehydrated through increasing concentration of sucrose solution (10\%, $20 \%, 30 \%)$. After embedding with Tissue-Tek OCT compound (4583; SAKURA, Torrance, CA, USA), the tissue was frozen in $-80^{\circ} \mathrm{C}$ or in liquid nitrogen for long-time preservation. Tissues were sliced into $8 \mu \mathrm{m}$. Slices were incubated with the following rat-specific primary antibodies: GFAP (bs-0199R, Bioss, China), NeuN (ab177487, Abcam, USA), Anti-CD31 antibody (ab24590, Abcam, USA), Anti-CD31 (bs-0195R, Bioss,China), ZO-1 (21773-1-AP, Proteintech, China), Claudin5 (35-2500, Invitrogen, USA), CD68 (ab31630, Abcam, USA), Iba1 (10904-1-AP, Proteintech, China), PDGFR2 (3169s, CST, USA). For visualization, fluorescent Alexa Fluor 488 Goat anti-Rabbit (a0423, Beyotime, China) or Cy3-labeled Goat Anti-Mouse (a0521, Beyotime, China) or fluorescent Alexa Fluor 488 Goat anti-Mouse (a0428, Beyotime, China) or Cy3-labeled Goat Anti-Rabbit (a0516, Beyotime, China) secondary antibodies were used for both or single staining at room temperature for $1 \mathrm{~h}$. Cell nuclei was visualized by Vectashield containing DAPI (ab104139, Abcam, USA). A Leica fluorescence microscope (Leica DMi8, Germany) was used to image and analysis.

\section{Western blot}

The expression of thrombin and downstream signaling proteins were quantified by western blot. Injured spinal cord epicenters $0.5 \mathrm{~cm}$ in size were harvested from three individual rats and lysed by homogenization in RIPA Lysis Buffer (P0013B, Beyotime) $(100 \mu \mathrm{L} / 10 \mathrm{mg}$ spinal cord) with PhosSTOP (04906837001; phosphatase inhibitor) and cOmplete (04693132001; protease inhibitor) from Roche (Mannheim, Germany). The protein concentration of each sample was measured using the bicinchoninic acid assay (BCA) (BL52A, biosharp, China). After SDS-PAGE and PVDF membrane transfer, antibodies were used to probe different proteins. Anti-thrombin (ab92621, Abcam, USA), anti-PAR1 (GTX64534, Gene Tex, USA), anti-MMP9 (ab38898, Abcam, USA), and anti-GAPDH (sc-32233, Santa Cruz Biotechnology, USA) were used. The blots were visualized using the Immobilon chemiluminescence system (Immobilon Western, Millipore, MA, USA). Image analysis was performed by ImageJ. Statistical comparisons of each protein were shown by the standard error and mean (SEM) across at least 3 individual animals.

\section{Statistical analyses}

Statistical analyses were conducted by GraphPad Prism 9.0 (San Diego, CA, USA). Data were presented as mean \pm SEM in figures with error bars. $P$ values $<0.05$ were considered statistically significant. T-test was used to compare two independent groups. One-way ANOVA with Tukey's or Dunnett's multiple comparisons post hoc test was used to compare more than two groups, and a two-way ANOVA with Bonferroni's post hoc test were used if two variables were independent.

\section{Results}


NM improved functional recovery notably at 0-12 $\mathrm{h}$ administration time post SCl

We demonstrated that intraperitoneal injection of $10 \mathrm{mg} / \mathrm{kg} / \mathrm{d}$ Nafamostat mesylate effectively improves functional recovery after $\mathrm{SCl}$ in rats[9], but the optimal administration time was still unclear. To accelerate the clinical transformation of NM, we designed a series of different first administration time (FAT) points to observe functional recovery (Fig. 1A). The FAT time was $0 \mathrm{~h}, 2 \mathrm{~h}, 8 \mathrm{~h}, 12 \mathrm{~h}, 24 \mathrm{~h}, 48 \mathrm{~h}$ and $5 \mathrm{~d}$ post injury followed by twice daily administration. After the operation, BBB scores were used to identify the locomotor functional recovery in different groups of rats weekly (Fig.1B). Significant improved hindlimb function recovery was observed at the first administration time of $0 \mathrm{~h}, 2 \mathrm{~h}, 8 \mathrm{~h}$ and $12 \mathrm{~h}$ postoperatively. Notably, most optimal functional recovery was achieved with $8 \mathrm{~h}$ and $12 \mathrm{~h}$ FAT. At the same time, we also detected the upstream and downstream of nerve conduction function at 5 weeks after $\mathrm{SCl}$ in rats by electrophysiology. We found that in both SEP and MEP, the amplitude was significantly higher after NM intervention ( $2 \mathrm{~h}, 8 \mathrm{~h}, 12 \mathrm{~h}$ ), especially in the group of $8 \mathrm{~h}$ and $12 \mathrm{~h}$. Meanwhile, the latency period after $\mathrm{NM}$ intervention at the time point of $0 \mathrm{~h}, 2 \mathrm{~h}, 8 \mathrm{~h}, 12 \mathrm{~h}$ was significantly shorter than that in $\mathrm{SCl}$ group. In conclusion, $\mathrm{NM}$ administration at 0 to $12 \mathrm{~h}$ after $\mathrm{SCl}$ can effectively improve the recovery of nerve conduction function in rats. Previously, we have proved that argatroban, a selective thrombin synthetic inhibitor, has a therapeutic effect on SCI[28]. Here, we used argatroban as a positive control, showing a similar time window against $\mathrm{SCl}$, which indicated that $\mathrm{NM}$ repairs SCl by thrombin inhibition (Fig. S1A, B). Based on the above several common functional recovery evaluation indicators, we widened the effective time window of NM intervention (0-12 h). To further confirm the optimal time window for NM administration, we performed a gait analysis by the Noldus CatWalk XT system on rats of the $8 \mathrm{~h}$ administration group at $5 \mathrm{w}$ (Fig. S2). Specifically, both NM (8 hpi) and argatroban (8 hpi) groups led to significant improvement in Cadence, Step Cycle and Stand of both hind limbs. However, only NM improved the $\mathrm{RI}(\%)$ after $\mathrm{SCl}$ significantly. This evidence indicated that the strength and coordination of the hindlimbs of rats after $\mathrm{SCl}$ were significantly enhanced after $\mathrm{NM}$ administration.

Together, we demonstrated that $0-12 \mathrm{~h}$ after $\mathrm{SCl}$ is the effective time window of NM intervention, which can notably improve the recovery of motor function, nerve conduction function and hind limb coordination after $\mathrm{SCl}$, especially at $8 \mathrm{~h}$ post injury.

\section{$\mathrm{NM}$ administration after $2 \mathrm{~h}$ post SCl did not affect the coagulation function.}

Given that inhibition of thrombin may increase the risk of clotting dysfunction, we also measured the clotting function at different FATs (Fig. S3). The results of prothrombin time (PT) (Fig. S3A), prothrombin activity (PTA) (Fig. S3B), and prothrombin time international normalized ratio (PT-INR) (Fig. S3C) reminded us that $0 \mathrm{~h}$ FAT group did show increased clotting dysfunction, while 2-12 h FAT groups revealed no risk of clotting disorders.

Together with behavioral evaluation, we indicated that 2-12 $\mathrm{h}$ after $\mathrm{SCl}$ is the safe and optimal time window of NM intervention to repair SCl.

\section{Thrombin temporal and spatial distribution pattern post SCI}


NM is a broad-spectrum serine protease inhibitor with thrombin as its main target[29]. To investigate the mechanism of $\mathrm{NM}$ in repairing $\mathrm{SCl}$, we discussed the distribution of thrombin and its downstream proteins post SCl (Fig. 2A). First, we first discussed the activity of thrombin in spinal cord (Fig. 2 B, C), finding that the thrombin activity presented a time-associated pattern. The activity of thrombin increased from $2 \mathrm{~h}$, peaked at $12 \mathrm{~h}$ post $\mathrm{SCl}$, and maintained a high expression level until $3 \mathrm{dpi}$. We also investigated the protein expression of thrombin, PAR1 and MMP9 during the acute $\mathrm{SCl}$ in the epicenter (Fig. 3D, E). Then, we questioned whether NM could inhibit thrombin and MMP9 in the spinal cord parenchyma (Figure. 2D). We detected the thrombin activity 3 dpi in the spinal cord epicenter (Fig. 2 E, F), finding that the thrombin activity was significantly inhibited by NM, which showed a similar inhibitory effect as Argatroban. Like the thrombin activity, at $2 \mathrm{~h}$ post SCl, the protein level of thrombin in epicenter increased about 3.75 -fold, peaked at $12 \mathrm{~h}(5.68$-fold $)$ and maintained a high level of expression until $3 \mathrm{dpi}$ (Fig. 2 G, H). A significant increase on PAR1 was also observed after $\mathrm{SCl}$ at the acute phase. Interestingly, the temporal expression pattern of MMP9 was followed by that of thrombin and PAR1, and the changes of MMP9 were more dramatic than that of thrombin. We indicated a 2-fold upregulation of MMP9 $2 \mathrm{~h}$ post injury with no statistical significance $(P=0.07)$. Gradually, the expression of MMP9 was sharply increased with a 10 -fold at $8 \mathrm{~h}$ and 20 -fold at $12 \mathrm{~h}$ post $\mathrm{SCl}$, and still maintained a high level until $3 \mathrm{dpi}$ which was the same with thrombin. According to the above data, we investigated that the effective time window of NM was completely consistent with the temporal expression of thrombin and MMP9 after SCl. Meanwhile, we also found a sharp decrease of thrombin expression after administration 3 dpi (Fig. $2 \mathrm{I}, \mathrm{J}$ ). And a decrease of MMP9 was found along with the inhibition of thrombin (Fig. $2 \mathrm{I}, \mathrm{K}$ ). These results indicated that NM inhibited thrombin activity and protein expression level, along with a decreased expression of MMP9.

\section{NM targeted the endothelial cells to inhibit the expression of thrombin and MMP9}

To further explore the mechanism of NM in SCl repair, we further investigated the spatial expression of thrombin (Fig. 3 A, Fig. S4). We found a basic expression of thrombin, which did not alter much after SCl in neurons, astrocytes and pericytes. Notably, we found that the greatest change of thrombin expression came from $\mathrm{CD} 31^{+} \mathrm{ECs}$ compared with the Sham group. And further, we also indicated that both $\mathrm{Iba} 1^{+}$ microglia and Olig2 ${ }^{+}$oligodendrocyte did not express thrombin before and post SCl. Together, these results demonstrated the temporal and spatial distribution in the epicenter of the spinal cord after SCl. We indicated that thrombin expression and activity peaked at $12 \mathrm{~h}$ temporally and thrombin expression was mainly concentrated in vascular ECs after spinal cord injury spatially.

Therefore, we further explored the changes of thrombin around vascular ECs after NM intervention. Immunofluorescence results showed that thrombin enrichment in vascular endothelial cells was significantly reduced. (Fig. 3 B, C). We also found that ECs expressed PAR1 abundantly, indicating that ECs can be regulated by thrombin (Fig. S5). The upregulated expression of MMP9 after SCl was also concentrated around the ECs, while both the expression of MMP9 and its close relationship with the blood vessels were inhibited after NM intervention (Fig. 3 D, E). 
Together, NM effectively inhibited the activity and expression of thrombin post $\mathrm{SCl}$, especially reduced the correlation between thrombin and vascular ECs. Meanwhile, MMP9 expression was also significantly inhibited.

\section{NM promoted blood-spinal cord barrier reconstruction post SCI}

We have demonstrated the inhibition effect of NM on thrombin, resulting in the reduction of MMP9, especially around ECs. Given that a high level of thrombin and MMP9 in the CNS resulted in a destructive BBB dysfunction[30], we asked whether NM regulated BSCB function by inhibiting thrombin and thus improved the outcome post SCI. To determine the function of BSCB, EB $(10 \mathrm{mg} / \mathrm{mL} / \mathrm{rat})$ was injected via tail vein (Fig. 4 A). After $3 \mathrm{~h}$-circulation in the body, the epicenter of the spinal cord was dissected. As shown (Fig. 4 B, C, Fig. S 6), at 1 dpi and 3 dpi, there was an obvious leakage of EB in the epicenter, suggesting severe damage of BSCB function in the acute $\mathrm{SCl}$. The leakage of EB was attenuated after the intervention of NM. At $1 \mathrm{dpi}, 2 \mathrm{~h}$ group had the most significant improvement of EB leakage, and there was also significant improvement in the group of $0 \mathrm{~h}, 8 \mathrm{~h}$ and $12 \mathrm{~h}$. While at $3 \mathrm{dpi}$, there was a significant decrease of EB leakage in the 0-12 $\mathrm{h}$ administration. Notably, at $7 \mathrm{dpi}$ (the subacute phase), no significant difference was observed between rats with or without NM intervention on EB leakage. Here, we proved that the repair time window of NM on BSCB permeability was completely consistent with the time window of functional recovery of $\mathrm{SCl}$, indicating the mechanism of $\mathrm{NM}$ repairing $\mathrm{SCl}$ involves its protective effect on BSCB.

\section{$\mathrm{NM}$ administration reduced the tight junction proteins breakdown post $\mathrm{SCl}$}

Among the components of BSCB, TJPs constitute the main mechanical barrier and charge barrier[31]. Since we found that increased BSCB permeability could be reversed by appropriate treatment of NM, we asked whether NM altered the expression of TJPs post SCI. We chose two representative TJPs, ZO-1 and CLD5 (Fig. 5 A, D). We first investigated the vessel intensity by CD31 after SCl, finding no significant difference between Sham, SCl, and NM group (Fig. 5 C, F). The coverage of ZO-1 and CLD5 in the epicenter of the spinal cord decreased significantly after $\mathrm{SCl}$, suggesting that many spinal vessels have no barrier function post SCl. And the decrease could be reversed by NM treatment 3 dpi (Fig5. D, G). These results indicated that NM declined the BSCB permeability by upregulating the expression of ZO-1 and CLD5.

\section{NM inhibited macrophage and neutrophil infiltration post SCI}

The inflammatory cascade in the acute $\mathrm{SCl}$ is mainly derived from peripheral inflammatory cells[32]. The infiltration of peripheral inflammatory cells after injury is attributed to the server destruction of BSCB. These inflammatory cells dramatically altered the microenvironment during the acute $\mathrm{SCl}$ and negatively affected long-term neurological outcome. Since we have investigated the protective effect of NM on the BSCB, we observed the infiltration of peripheral macrophage and neutrophil 3 dpi (Fig. 6 A, D). We found a significant decrease in the number of infiltrating $\mathrm{CD}^{+} 8^{+}$macrophage and $\mathrm{MPO}^{+}$neutrophil in the spinal cord parenchyma in the NM group compared with the SCI group (Fig6 C, E). These results suggested that 
NM could prevent the infiltration of peripheral macrophage and neutrophil into parenchyma to some extent after reducing the permeability of BSCB.

\section{Discussion}

NM is an FDA-approved drug in cure of pancreatitis. There is an expanded effect in other diseases, such as COVID-19[33]. We have previously reported that NM has beneficial effect on SCI[34, 35]. However, its administration time window and specific mechanism remain unclear. In this study, we successfully proved the effective administration time window of NM after SCl and the medication-related safety. Mechanically, NM repaired SCl through inhibiting the expression and activity of thrombin, and protected ECs from the activation of PAR1 on its surface, then prevented neutrophil and macrophage infiltration and reduced MMP9 release, thereby preventing TJPs degradation, and protected BSCB to repair SCI.

$\mathrm{MP}$ is currently the only FDA-approved drug for acute SCI treatment[36]. Although several clinical trials supported that MP should be used within $8 \mathrm{~h}$ post injury, and the duration should not exceed $24 \mathrm{~h}$, there have been many criticisms of MP in recent years because of increasing incidence of complications[3]. Here, we selected NM as a candidate to solve the above questions in current study as a safe, effective, and broader time-window drug for acute $\mathrm{SCl}$ repair. 6 different initial FATs were used to evaluate the optimal administration time window for $\mathrm{NM}$ to repair $\mathrm{SCl}$, we observed that administration between immediately and $12 \mathrm{~h}$ after SCl could effectively promote the recovery of long-term locomotor and nerve conduction function. Since NM has a certain anticoagulant effect, we also tested the peripheral blood coagulation function and proved the safety of the administration starting $2 \mathrm{~h}$ after SCI[9]. In terms of overall safety and effectiveness, we determined $2 \mathrm{~h}$ to $12 \mathrm{~h}$ to be the optimal time window for administration. In conclusion, compared with MP, the advantages of NM for early intervention are obvious, including wider administration time window and lower side effects, which is more suitable for clinical application.

Here, we also used argatroban, a direct inhibitor of thrombin, as a positive control. However, we discovered that it had less curative effect than that of NM, which may be attributed that NM could inhibit the serine protease system in the microenvironment of SCl. Therefore, we believed that it is necessary to further study the role of the whole serine protease system in SCl.

Thrombin is a trypsin-like serine protease and is the most abundant enzyme involved in the coagulation cascade[37]. In addition to hemostasis, thrombin also regulates a variety of pathogenesis of CNS disease through BBB dysfunction. MMP9 is upregulated during ischemic stroke[38], intracerebral hemorrhage (ICH)[39], and SCI[40]. Studies have shown that thrombin and MMP9 are very important in the pathogenesis of neurovascular diseases with BBB dysfunction and have a synergistic effect in ICH to aggravate damage[41, 42]. Our study showed that the temporal distribution pattern of thrombin, PAR1 and MMP9 after $\mathrm{SCl}$ is similar, and the peak value appears at about $12 \mathrm{~h}$ after injury. At the same time, thrombin activity also peaks at $12 \mathrm{~h}$ after injury, which is consistent with its expression level. Inhibiting their expression and activity before the peak will promise an effective role in hindering the development 
of secondary SCI. In administration time window research, we chose 8-12 h after SCl was the optimal administration, which happened to be the peak of thrombin and MMP9. Accordingly, we further proved the rationality of the administration time window from the molecular level. MMP9 expression was increased dramatically through thrombin activated PAR1 after $\mathrm{SCl}$, further aggravating TJPs degradation, and exacerbated the destruction of BSCB and inflammatory cells in peripheral blood infiltrated into the injured spinal cord[43].

BSCB is a physiological barrier between blood and spinal cord parenchyma, composed of ECs, astrocyte ends, pericyte, as well as basal layer and tight/adhesive junction proteins[44]. The BSCB breakdown after $\mathrm{SCl}$ accelerates the exchange of substances between the spinal cord microenvironment and the circulating blood, resulting in a microenvironmental imbalance[45]. Chemokines attract amount of inflammatory cells and pro-inflammatory factors in the serum to the injured spinal cord, leading to an inflammatory cascade[46]. We found that ECs express thrombin receptor PAR1. Moreover, large amount of thrombin accumulated around $\mathrm{CD} 31^{+}$vascular ECs, and the administration of NM reduced the expression and activity of thrombin. Meanwhile, MMP9 expression around the vascular ECs decreased significantly after NM intervention, indicating that the activation of PAR1 is a critical factor leading to the loss of TJPs and the BSCB breakdown. We believe that the dramatic increase in thrombin concentration after $\mathrm{SCl}$ activates PAR1 and upregulation of MMP9 expression, which induced the destruction of the BSCB. While NM protects the BSCB by inhibiting thrombin activity, thus regulating the imbalance of spinal cord microenvironment after SCl.

Here, we proved that there was no significant difference in BSCB permeability between the groups $7 \mathrm{~d}$ after $\mathrm{SCl}$ regardless of treatment by EB staining, indicating that $\mathrm{BSCB}$ could spontaneously recover during the subacute $\mathrm{SCl}$. However, most of the secondary pathological processes after $\mathrm{SCl}$ occurred in the acute phase (0-3 dpi)[47], and the protective effect of the BSCB during the acute phase is beneficial for longterm recovery. In this study, 2-12 $\mathrm{h}$ administration can effectively protect BSCB from breakdown through inhibiting thrombin and MMP9. Administration of NM at $\mathrm{O}$ h after injury may promote local bleeding in the injury epicenter to a certain extent, resulting in a slightly worse degree of BSCB breakdown than other treatment groups at $1 \mathrm{~d}$ after injury, which once again verified the rationality of the administration time window.

In our previous study, the result of inflammation chip sequencing showed that NM could reduce many chemokines expression in local site of SCI[34]. Here, we also proved that administration of NM after SCI reduced neutrophil and macrophage infiltration significantly, which we believed that the protection of BSCB was a considerable mechanism of the anti-neuroinflammation effect induced by NM.

\section{Conclusion}

Our study provided preclinical data of NM administration time window in SCI model for the first time, which is clinically relevant in the acute $\mathrm{SCl}$ (Figure. 7). We elucidated the administration of NM can be 
effective in inhibiting thrombin/PAR1/MMP9 axis, protecting ECs and TJPs, reducing infiltration of inflammatory cells and neuroinflammation, thereby repairing SCl.

\section{Abbreviations}

\begin{tabular}{ll} 
NM & nafamostat mesylate \\
\hline SCI & spinal cord injury \\
\hline PAR-1 & protease activated receptor 1 \\
\hline MMP9 & matrix metalloproteinase 9 \\
\hline ECs & endothelial cells \\
\hline BSCB & blood-spinal cord barrier \\
\hline TJPs & tight junction proteins \\
\hline MP & methylprednisolone \\
\hline CNS & central nervous system \\
\hline BBB & blood brain barrier \\
\hline BSCB & blood spinal cord barrier \\
\hline TBI & traumatic brain injury \\
\hline ZO-1 & zonulaoccludens-1 \\
\hline CLD5 & Claudin5 \\
\hline FAT & first administration time \\
\hline RI & regularity index \\
\hline SEP & sensory evoked potential \\
\hline MEP & motor evoked potential \\
\hline PT & prothrombin time \\
\hline PTA & prothrombin activity \\
\hline INR & international normalized ratio \\
\hline EB & Evans Blue \\
\hline SEM & standard error and mean \\
\hline S
\end{tabular}

\section{Declarations}


Not applicable.

\section{Authors' contributions}

$Y X, Z C$, and ZT designed the experiments. ZC, ZT and ZX contributed to the major acquisition of data and did the analysis and interpretation of data. YX, ZC, ZT and ZX drafted the manuscript. PY, LW, LM, LX, ML and ZJW helped with the animal experiments. FB, LX, ZJ, SW and KX discussed the experimental results and reviewed the article. FS and YX conceived the hypothesis, supervised the study, and provided the funds. All authors read and approved the final manuscript.

\section{Funding}

We thank the support of the following funding: Key project of National Natural Science Foundation of China [81930070], National Key Research and Development Project [2019YFA0112100], National Natural Science Foundation of China program [81972074], Key Program of Natural Science Foundation of Tianjin [19JCZDJC34900].

\section{Availability of data and materials}

The data obtained from this study are included in the article or the supplementary files.

\section{Ethics approval and consent to participate}

Animal studies were approved by the Ethics Committee of Tianjin Medical University (Tianjin, China, IRMDWLL-2020080).

\section{Consent for publication}

Not applicable.

\section{Competing interests}

The authors declare that they have no competing interests.

\section{References}

1. Badhiwala, J.H., C.S. Ahuja, and M.G. Fehlings, Time is spine: a review of translational advances in spinal cord injury. J Neurosurg Spine, 2018. 30(1): p. 1-18.

2. Nagoshi, N. and M.G. Fehlings, Investigational drugs for the treatment of spinal cord injury: review of preclinical studies and evaluation of clinical trials from Phase I to II. Expert Opin Investig Drugs, 2015. 24(5): p. 645-58.

3. Bracken, M.B., et al., Administration of methylprednisolone for 24 or 48 hours or tirilazad mesylate for 48 hours in the treatment of acute spinal cord injury. Results of the Third National Acute Spinal 
Cord Injury Randomized Controlled Trial. National Acute Spinal Cord Injury Study. JAMA, 1997. 277(20): p. 1597-604.

4. Griffin, J.M. and F. Bradke, Therapeutic repair for spinal cord injury: combinatory approaches to address a multifaceted problem. EMBO Mol Med, 2020. 12(3): p. e11505.

5. Fujii, S. and Y. Hitomi, New synthetic inhibitors of C1r, C1 esterase, thrombin, plasmin, kallikrein and trypsin. Biochim Biophys Acta, 1981. 661(2): p. 342-5.

6. Chen, T., et al., Nafamostat mesilate attenuates neuronal damage in a rat model of transient focal cerebral ischemia through thrombin inhibition. Sci Rep, 2014. 4: p. 5531.

7. Fuwa, M., et al., Nafamostat and sepimostat identified as novel neuroprotective agents via NR2B Nmethyl-D-aspartate receptor antagonism using a rat retinal excitotoxicity model. Sci Rep, 2019. 9(1): p. 20409.

8. Kwon, S.K., et al., Nafamostat mesilate attenuates transient focal ischemia/reperfusion-induced brain injury via the inhibition of endoplasmic reticulum stress. Brain Res, 2015. 1627: p. 12-20.

9. Duan, H.Q., et al., Nafamostat mesilate attenuates inflammation and apoptosis and promotes locomotor recovery after spinal cord injury. CNS Neurosci Ther, 2018. 24(5): p. 429-438.

10. Beladi, R.N., et al., Serine Proteases and Chemokines in Neurotrauma; New Targets for Immune Modulating Therapeutics in Spinal Cord Injury. Curr Neuropharmacol, 2021.

11. Ebrahimi, S., et al., Role of thrombin in the pathogenesis of central nervous system inflammatory diseases. J Cell Physiol, 2017. 232(3): p. 482-485.

12. Ben Shimon, M., et al., Thrombin as Key Mediator of Seizure Development Following Traumatic Brain Injury. Front Pharmacol, 2019. 10: p. 1532.

13. Kim, H.N., et al., The thrombin receptor modulates astroglia-neuron trophic coupling and neural repair after spinal cord injury. Glia, 2021. 69(9): p. 2111-2132.

14. Radulovic, M., et al., Kallikrein cascades in traumatic spinal cord injury: in vitro evidence for roles in axonopathy and neuron degeneration. J Neuropathol Exp Neurol, 2013. 72(11): p. 1072-89.

15. Sinha, R.K., et al., PAR1 biased signaling is required for activated protein $C$ in vivo benefits in sepsis and stroke. Blood, 2018. 131(11): p. 1163-1171.

16. Machida, T., et al., Role of thrombin-PAR1-PKCtheta/delta axis in brain pericytes in thrombin-induced MMP-9 production and blood-brain barrier dysfunction in vitro. Neuroscience, 2017. 350: p. 146-157.

17. Lee, J.Y., et al., Valproic acid attenuates blood-spinal cord barrier disruption by inhibiting matrix metalloprotease-9 activity and improves functional recovery after spinal cord injury. J Neurochem, 2012. 121(5): p. 818-29.

18. Bartanusz, V., et al., The blood-spinal cord barrier: morphology and clinical implications. Ann Neurol, 2011. 70(2): p. 194-206.

19. Lee, J.Y., et al., Jmjd3 mediates blood-spinal cord barrier disruption after spinal cord injury by regulating MMP-3 and MMP-9 expressions. Neurobiol Dis, 2016. 95: p. 66-81. 
20. Bowes, A.L. and P.K. Yip, Modulating inflammatory cell responses to spinal cord injury: all in good time. J Neurotrauma, 2014. 31(21): p. 1753-66.

21. Davies, J.E., et al., Decorin promotes plasminogen/plasmin expression within acute spinal cord injuries and by adult microglia in vitro. J Neurotrauma, 2006. 23(3-4): p. 397-408.

22. Chen, B., et al., Thrombin mediates severe neurovascular injury during ischemia. Stroke, 2010. 41(10): p. 2348-52.

23. Basso, D.M., M.S. Beattie, and J.C. Bresnahan, $A$ sensitive and reliable locomotor rating scale for open field testing in rats. J Neurotrauma, 1995. 12(1): p. 1-21.

24. Dias, D.O., et al., Reducing Pericyte-Derived Scarring Promotes Recovery after Spinal Cord Injury. Cell, 2018. 173(1): p. 153-165 e22.

25. Yao, X., et al., Neurotropin exerts neuroprotective effects after spinal cord injury by inhibiting apoptosis and modulating cytokines. J Orthop Translat, 2021. 26: p. 74-83.

26. Ye, L.B., et al., Regulation of Caveolin-1 and Junction Proteins by bFGF Contributes to the Integrity of Blood-Spinal Cord Barrier and Functional Recovery. Neurotherapeutics, 2016. 13(4): p. 844-858.

27. $\mathrm{Pi}, \mathrm{X}$., et al., Prevalence and clinical characteristics of active epilepsy in southern Han Chinese. Seizure, 2014. 23(8): p. 636-40.

28. Zhao, C., et al., Role of argatroban in repair of spinal cord injury in rats. Chinese Journal of Trauma, 2020(01): p. 86-92.

29. Aoyama, T., et al., Pharmacological studies of FUT-175, nafamstat mesilate. I. Inhibition of protease activity in in vitro and in vivo experiments. Jpn J Pharmacol, 1984. 35(3): p. 203-27.

30. Rempe, R.G., A.M.S. Hartz, and B. Bauer, Matrix metalloproteinases in the brain and blood-brain barrier: Versatile breakers and makers. J Cereb Blood Flow Metab, 2016. 36(9): p. 1481-507.

31. Abdullahi, W., D. Tripathi, and P.T. Ronaldson, Blood-brain barrier dysfunction in ischemic stroke: targeting tight junctions and transporters for vascular protection. Am J Physiol Cell Physiol, 2018. 315(3): p. C343-C356.

32. Fan, B., et al., Microenvironment Imbalance of Spinal Cord Injury. Cell Transplant, 2018. 27(6): p. 853866.

33. Sivaraman, H., et al., Structural Basis of SARS-CoV-2- and SARS-CoV-Receptor Binding and SmallMolecule Blockers as Potential Therapeutics. Annu Rev Pharmacol Toxicol, 2021. 61: p. 465-493.

34. Sun, C., et al., Cytokine expressions of spinal cord injury treated by neurotropin and nafamostat mesylate. Ann Transl Med, 2021. 9(6): p. 489.

35. Duan, H.-Q., et al., Nafamostat mesilate attenuates inflammation and apoptosis and promotes locomotor recovery after spinal cord injury. CNS neuroscience \& therapeutics, 2018. 24(5): p. 429438.

36. Breslin, K. and D. Agrawal, The use of methylprednisolone in acute spinal cord injury: a review of the evidence, controversies, and recommendations. Pediatr Emerg Care, 2012. 28(11): p. 1238-45; quiz $1246-8$. 
37. Dahlback, B., Blood coagulation. Lancet, 2000. 355(9215): p. 1627-32.

38. Turner, R.J. and F.R. Sharp, Implications of MMP9 for Blood Brain Barrier Disruption and Hemorrhagic Transformation Following Ischemic Stroke. Front Cell Neurosci, 2016. 10: p. 56.

39. Han, D., et al., Effect of Propofol on the Expression of MMP-9 and Its Relevant Inflammatory Factors in Brain of Rat with Intracerebral Hemorrhage. Cell Biochem Biophys, 2015. 72(3): p. 675-9.

40. Yu, F., et al., Induction of mmp-9 expression and endothelial injury by oxidative stress after spinal cord injury. J Neurotrauma, 2008. 25(3): p. 184-95.

41. Song, Y., et al., Lipoxin A4 Methyl Ester Reduces Early Brain Injury by Inhibition of the Nuclear Factor Kappa B (NF-kappaB)-Dependent Matrix Metallopeptidase 9 (MMP-9) Pathway in a Rat Model of Intracerebral Hemorrhage. Med Sci Monit, 2019. 25: p. 1838-1847.

42. Wu, G., et al., Early stage minimally invasive procedures reduce perihematomal MMP-9 and bloodbrain barrier disruption in a rabbit model of intracerebral hemorrhage. Neurol Res, 2013. 35(6): p. 649-58.

43. Lee, J.Y., et al., Fluoxetine inhibits matrix metalloprotease activation and prevents disruption of blood-spinal cord barrier after spinal cord injury. Brain, 2012. 135(Pt 8): p. 2375-89.

44. Jin, L.Y., et al., Blood-Spinal Cord Barrier in Spinal Cord Injury: A Review. J Neurotrauma, 2021. 38(9): p. 1203-1224.

45. Fan, B., et al., Microenvironment Imbalance of Spinal Cord Injury. Cell transplantation, 2018. 27(6): p. 853-866.

46. Anwar, M.A., T.S. Al Shehabi, and A.H. Eid, Inflammogenesis of Secondary Spinal Cord Injury. Front Cell Neurosci, 2016. 10: p. 98.

47. Lipinski, M.M., et al., Function and Mechanisms of Autophagy in Brain and Spinal Cord Trauma. Antioxid Redox Signal, 2015. 23(6): p. 565-77.

\section{Figures}



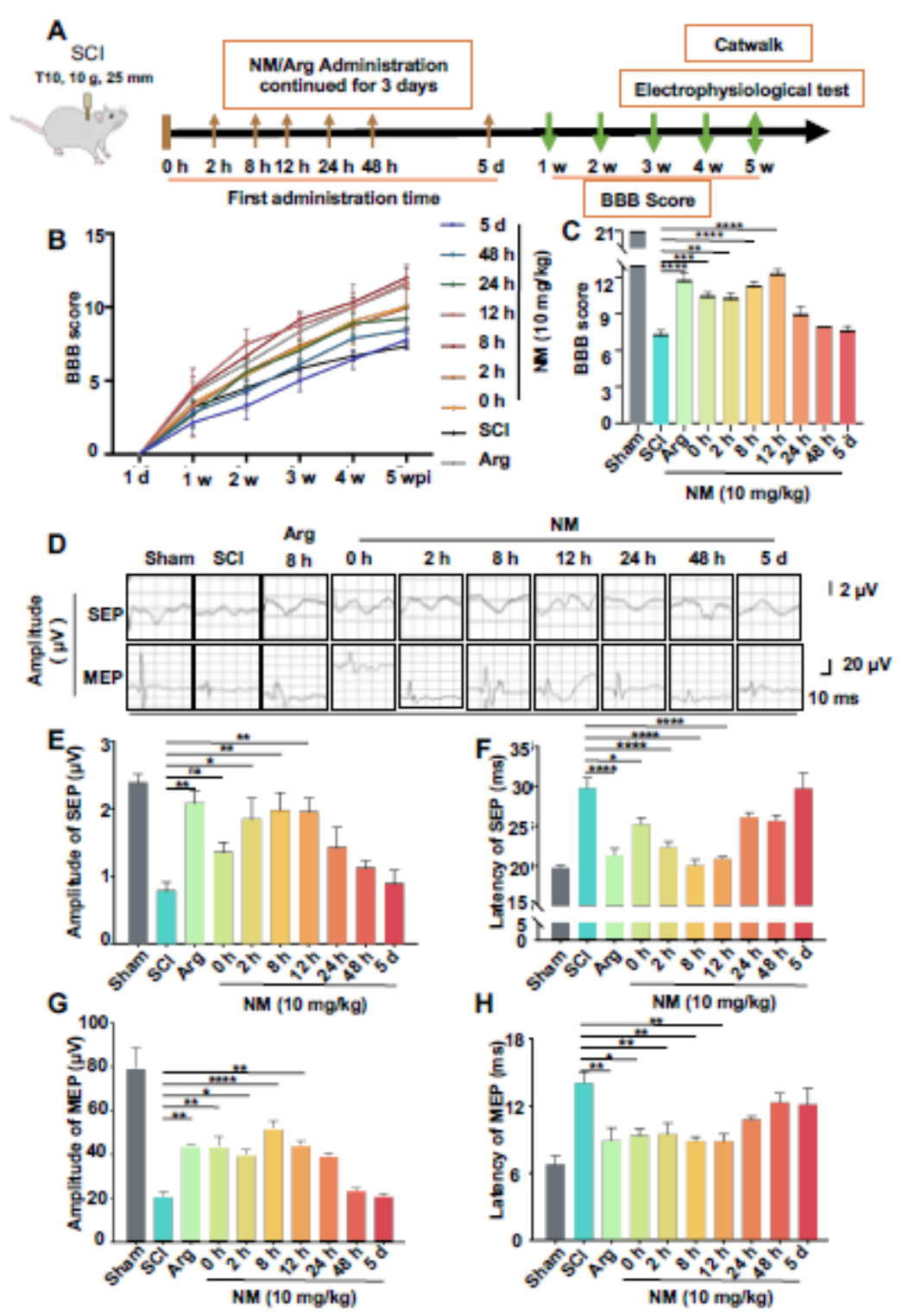

\section{Figure 1}

NM improved functional recovery notably at 0-12 $\mathrm{h}$ administration time after $\mathrm{SCl}(\mathrm{A})$. Illustration describing the experiment design for long-term experiments and motor function recovery. (B). The degree of hind-limb recovery was assessed for 5 weeks after SCI by BBB score. 7 different FATs of Nafamostat were 0 h, 2 h, 8 h, 12 h, 24 h, 48 h and 5 dpi, and FAT of Argatroban (Arg) was 8 hpi. (Data shown as mean \pm SEM, two-way ANOVA with Tukey's post-hoc test, ${ }^{*} P<0.05,{ }^{*} \mathrm{P}<0.01,{ }^{*} * \mathrm{P}<0.001 \mathrm{vs}$. the $\mathrm{SCl}$ group, $n=6)(C)$. Comparison of BBB scores of each group at 5 week point post injury. (Data shown as mean \pm SEM, one-way ANOVA with Tukey's post-hoc test, ${ }^{*} P<0.05$, ${ }^{*} \mathrm{P}<0.01,{ }^{*} * \mathrm{P}<0.001$ vs. the $\mathrm{SCl}$ group, $\mathrm{n}=6$ ) (D). Representative SEP and MEP waveform of nerve electrophysiology examination of rats in each group at 5 weeks post $S C I$. $(E, F, G, H)$. Quantification of the amplitude and latency of SEP and MEP in each group at $5 \mathrm{w}$ post $\mathrm{SCl}$. (Data shown as mean $\pm \mathrm{SEM}$, one-way ANOVA with Tukey's post-hoc test, ${ }^{*} P<0.05$, ${ }^{* *} \mathrm{P}<0.01,{ }^{* \star *} \mathrm{P}<0.001$ vs. the $\mathrm{SCl}$ group, $\mathrm{n}=6$ ) 

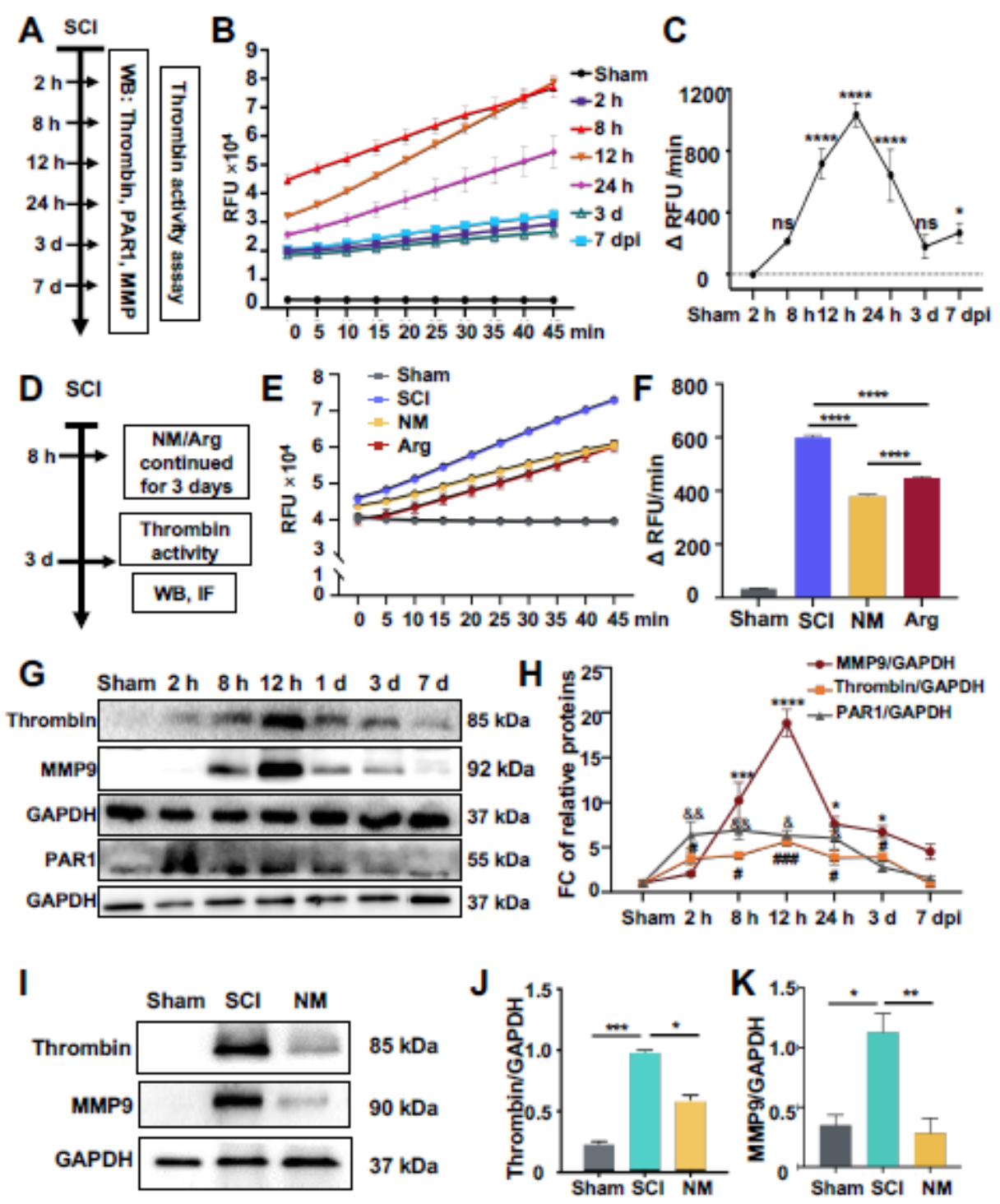

Figure 2

NM inhibited the expression of thrombin and MMP9 after SCl in the injured spinal cord. (A). Illustration describing the experiment design for temporal expression of thrombin and its downstream proteins. (B). Representative line chart of temporal thrombin activity after $\mathrm{SCl}(2 \mathrm{~h}, 8 \mathrm{~h}, 12 \mathrm{~h}, 24 \mathrm{~h}, 3 \mathrm{~d}, 7 \mathrm{~d})$. (C). Quantitively analysis of thrombin activity. (Data shown as mean \pm SEM, one-way ANOVA with Tukey's post-hoc test, $\left.{ }^{*} P<0.05,{ }^{*} P<0.01,{ }^{*} * P<0.001, n=3\right)(D)$. Illustration describing the experiment design for the inhibition of thrombin and its downstream proteins. (E). Representative line chart of thrombin activity 3 days after $\mathrm{SCl}$. (F). Quantitively analysis of thrombin activity. (Data shown as mean $\pm \mathrm{SEM}$, one-way ANOVA with Tukey's post-hoc test, $\left.{ }^{\star \star * *} \mathrm{P}<0.0001, \mathrm{n}=5\right)(\mathrm{G})$. Representative western blot image of the temporal expression after SCl $(2 \mathrm{~h}, 8 \mathrm{~h}, 12 \mathrm{~h}, 24 \mathrm{~h}, 3 \mathrm{~d}, 7 \mathrm{~d})$ of thrombin, PAR 1 and MMP9. The expression level of each protein was normalized by GAPDH. $(\mathrm{H})$. Quantitively analysis of the temporal expression of MMP9, thrombin and PAR1 normalized by GAPDH. (Data shown as mean \pm SEM, one-way ANOVA with Tukey's post-hoc test, $P$ value of MMP9 was shown as *, thrombin as \#, and PAR1 as $\&,{ }^{*} P<0.05$, $* * P<$ $0.01,{ }^{\star}{ }^{*} \mathrm{P}<0.001$ vs. the $\mathrm{SCl}$ group, $\left.\mathrm{n}=3\right)(\mathrm{I})$. Representative western blot image of thrombin and MMP 
expression 3 days after SCl. (J, K). Quantitively analysis of thrombin and MMP9 expression level. (Data shown as mean $\pm S E M$, unpaired t-test, ${ }^{*} P<0.05, * * P<0.01$, $* \star * P<0.001, n=3$ )
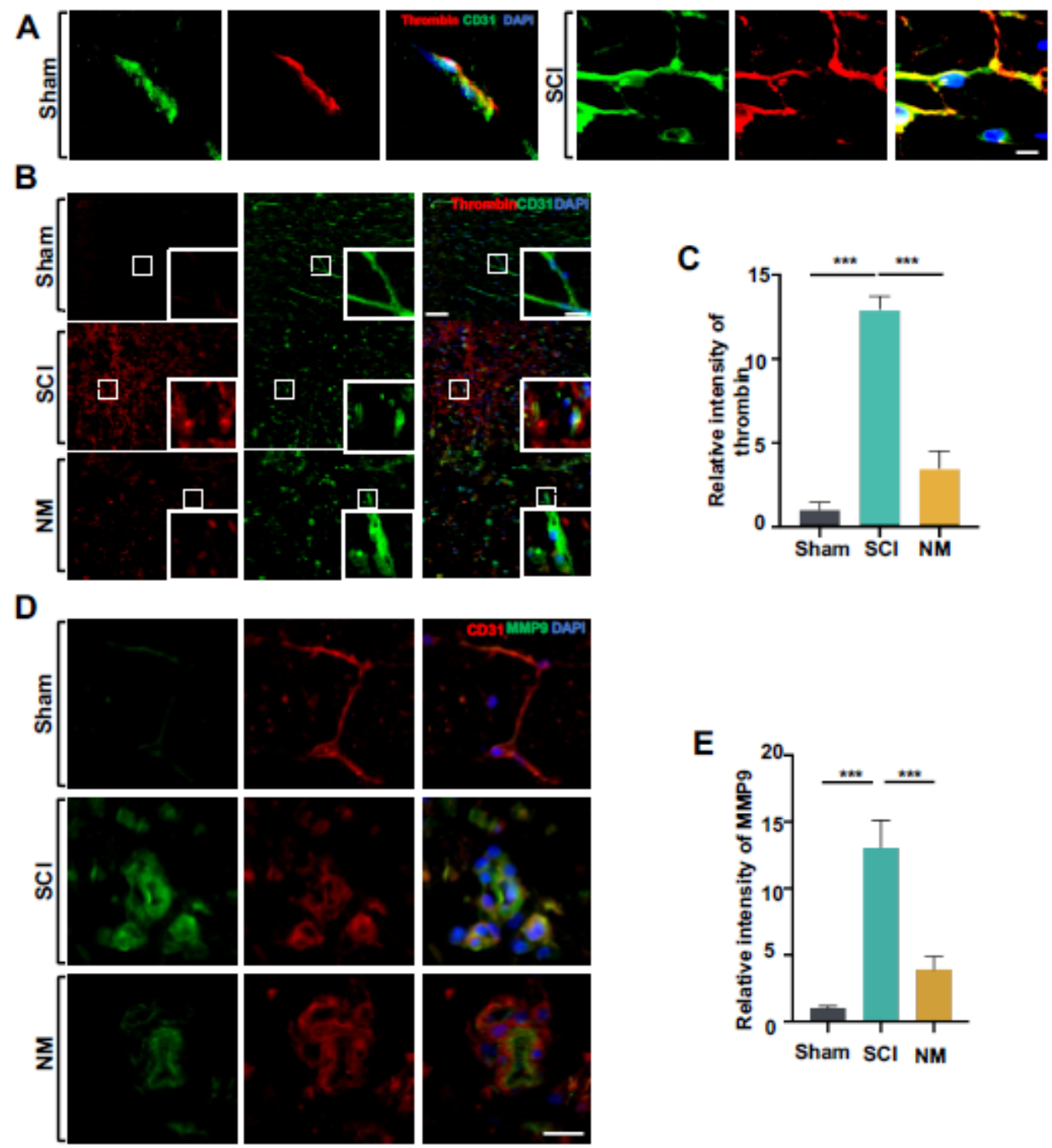

\section{Figure 3}

Nafamostat mesylate targeted the endothelial cells to inhibit the expression of thrombin and MMP9. (A). Representative co-localized images of thrombin (red) endothelial cell (CD31) at 3 days post injury. Cell nuclei were stained with DAPI (blue). Scale bar= $50 \mu \mathrm{m}$. (B). Immunofluorescence image of thrombin (red) and CD31 (green) at 3 days post injury. Cell nuclei were stained with DAPI (blue). Scale bar= $100 \mu \mathrm{m}$, $25 \mu \mathrm{m}$. (C). Quantitively analysis of the relative fluoresce intensity of thrombin. (Data shown as mean \pm SEM, one-way ANOVA with Tukey's post-hoc test, $\left.{ }^{\star * *} P<0.001, n=3\right)(D)$. Immunofluorescence image of CD31 (red) and MMP9 (green). Cell nuclei were stained with DAPI (blue). Scale bar= $10 \mu \mathrm{m}$. (E). Quantitively analysis of the relative fluoresce intensity of MMP9. (Data shown as mean \pm SEM, one-way ANOVA with Tukey's post-hoc test, ${ }^{\star} * * P<0.001, n=3$ ) 


\section{A}

$\mathrm{T} 10,10 \mathrm{~g}, 25 \mathrm{~mm}$
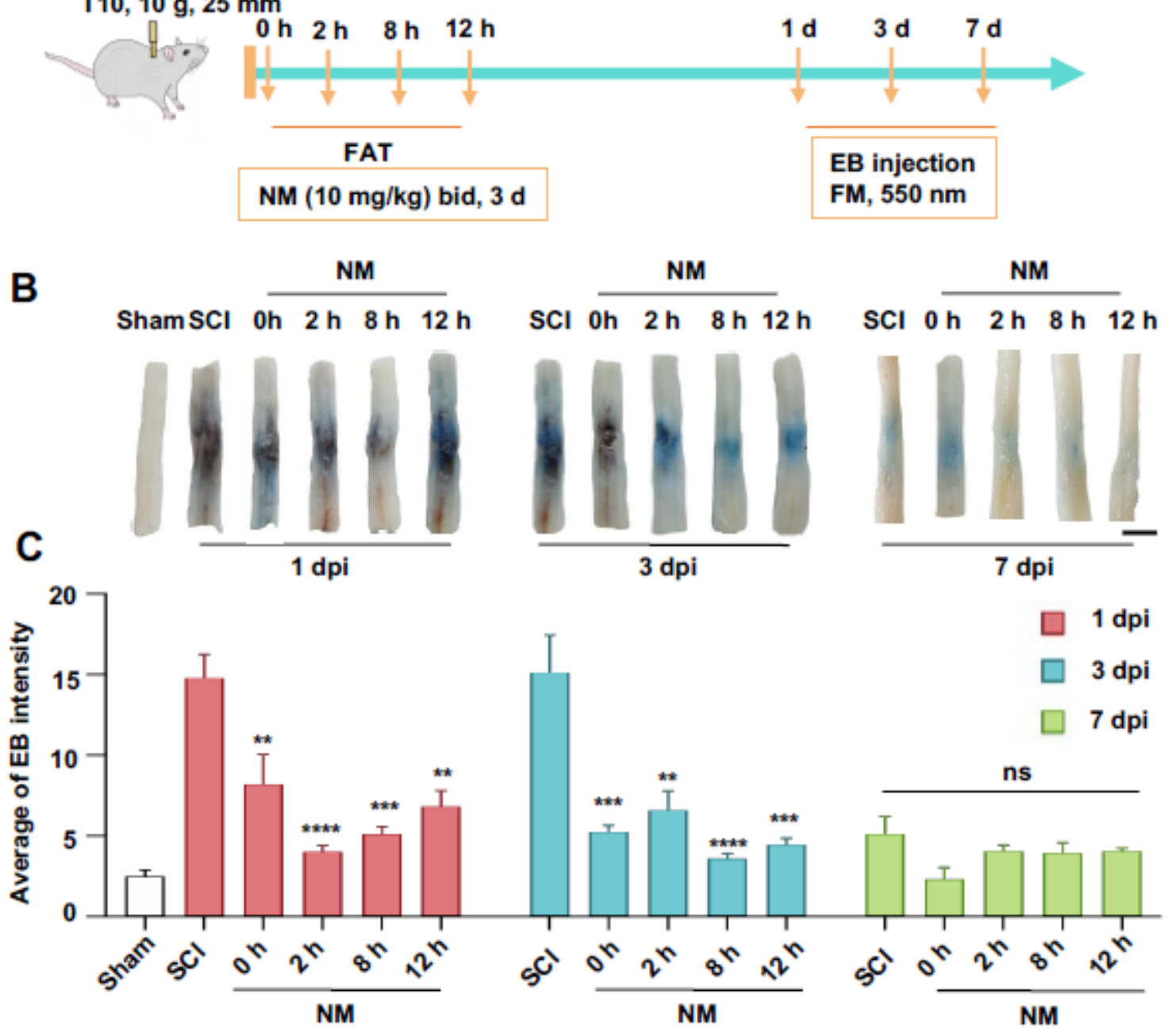

\section{Figure 4}

Nafamostat mesylate promotes blood-spinal cord barrier recovery after spinal cord injury in rats. (A). Illustration describing the experiment design for detection of the permeability of BSCB by Evans Blue (EB) leakage. After different FAT treatment of NM $(10 \mathrm{mg} / \mathrm{kg}$, bid) for 3 days after SCl, $1 \mathrm{ml} \mathrm{EB}(10 \mathrm{mg} / \mathrm{ml})$ was injected by tail vein. EB solution was allowed to circulate for $3 \mathrm{~h}$ before rats were sacrificed. EB leakage was detected using fluorescence microscopy (FM) under the excitation of $550 \mathrm{~nm}$ wave-length light. (B)Representative photos of general histogram of EB leakage after $\mathrm{SCl}$ at $1 \mathrm{~d}, 3 \mathrm{~d}, 7 \mathrm{~d}$ post injury of first administration, respectively. Scale bar $=1 \mathrm{~cm}$. (C). Quantitate analysis of average fluorescence intensity of Evans blue in spinal cord. (Data shown as mean \pm SEM, one-way ANOVA with Tukey's post-hoc test, $* * P<$ $0.01, \star \star \star P<0.001$ vs. the $S C l$ group, $n=4$ ) 

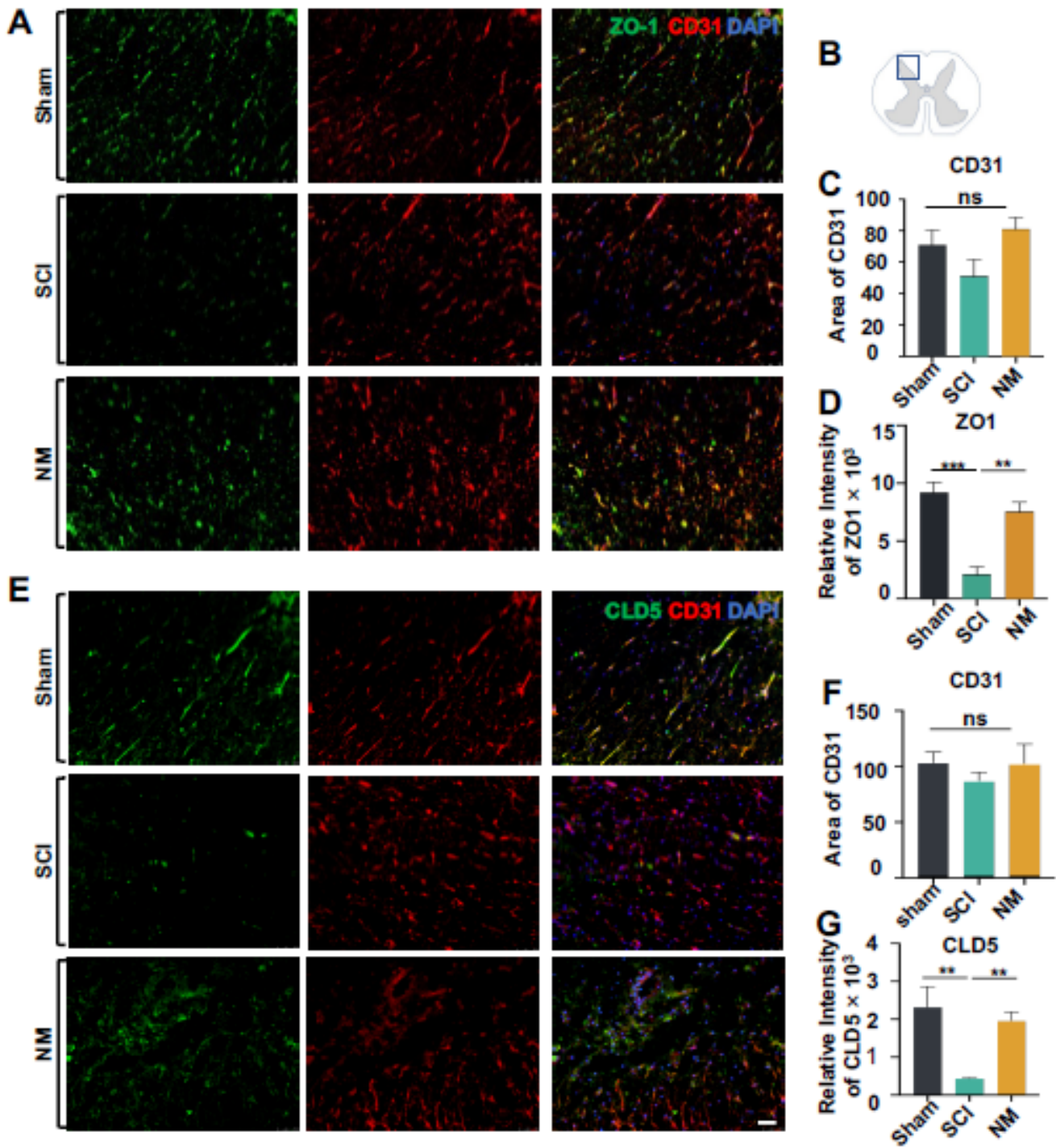

Figure 5

Nafamostat mesylate upregulated the expression of tight junction proteins after spinal cord injury. (A). Immunofluorescence image of CD31 (red) and ZO-1 (green) expression in posterior horn of spinal cord 3 days post injury. Scale bar=50 $\mu \mathrm{m}$. (B). Illustration describing the Image acquisition area in this figure. Fluorescence was acquisition at the posterior horn of the spinal cord. (C, D). Quantitively analysis of the area of CD31 and the relative intensity of ZO-1. (E). Immunofluorescence image of CD31 (red) and CLD5 (green) expression in posterior horn of spinal cord after 3 days post injury. Scale bar $=50 \mu \mathrm{m} .(F, G)$.

Quantitively analysis of the area of CD31 and the relative intensity of CLD5. Cell nuclei were stained with

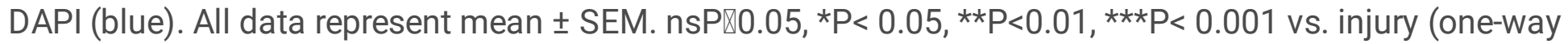
ANOVA with Tukey's post-hoc test), $\mathrm{n}=3$. 

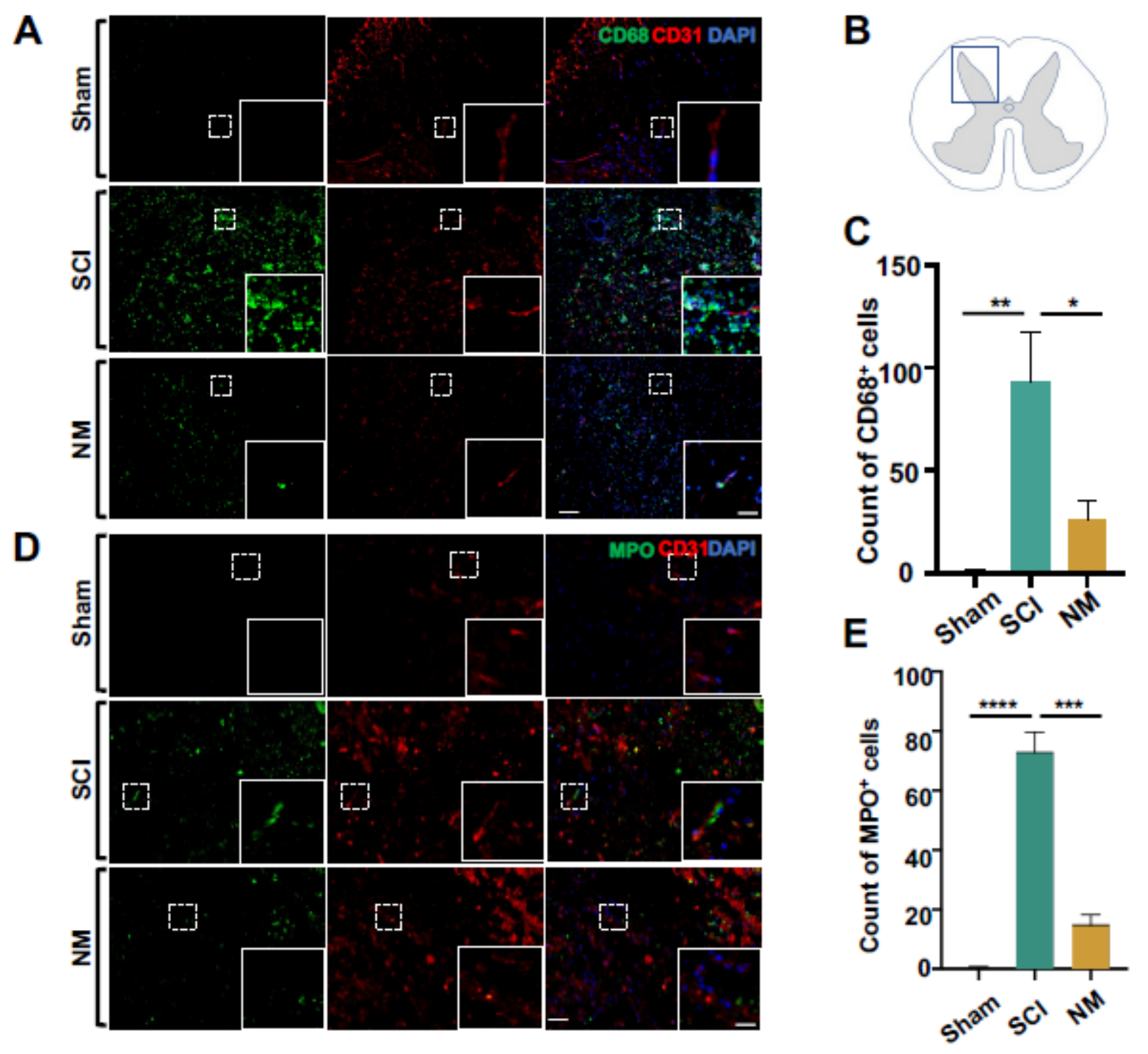

\section{Figure 6}

Nafamostat mesylate inhibited macrophage and neutrophil infiltration after $\mathrm{SCl}(\mathrm{A})$. Immunofluorescence image of CD31 (red) and CD68 (green) in posterior horn of spinal cord 3 days post injury. Cell nuclei were stained with DAPI (blue). Scale bar=50 $\mu \mathrm{m}$. (B). Illustration describing the Image acquisition area in this figure. Fluorescence was acquisition at the posterior horn of the spinal cord. (C). Quantitively analysis of the amount of $\mathrm{CD} 68+$ cells in posterior horn of spinal cord 3 days post injury. All data represent mean \pm SEM. ${ }^{*} P<0.05$ and ${ }^{*} P<0.01$ vs. injury (one-way ANOVA with Tukey's post-hoc test, $n=3$ ). (D). Immunofluorescence image of CD31 (red) and MPO (green) in posterior horn of spinal cord 3 days post injury. Cell nuclei were stained with DAPI (blue). Scale bar=50 $\mu \mathrm{m}$. (E). Quantitively analysis of the amount of MPO+ cells in posterior horn of spinal cord 3 days post injury. All data represent mean \pm SEM. ${ }^{\star} * \star P<0.001,{ }^{* \star * *} P<0.0001$ vs injury (one-way ANOVA with Tukey's post-hoc test, $n=3$ ). 


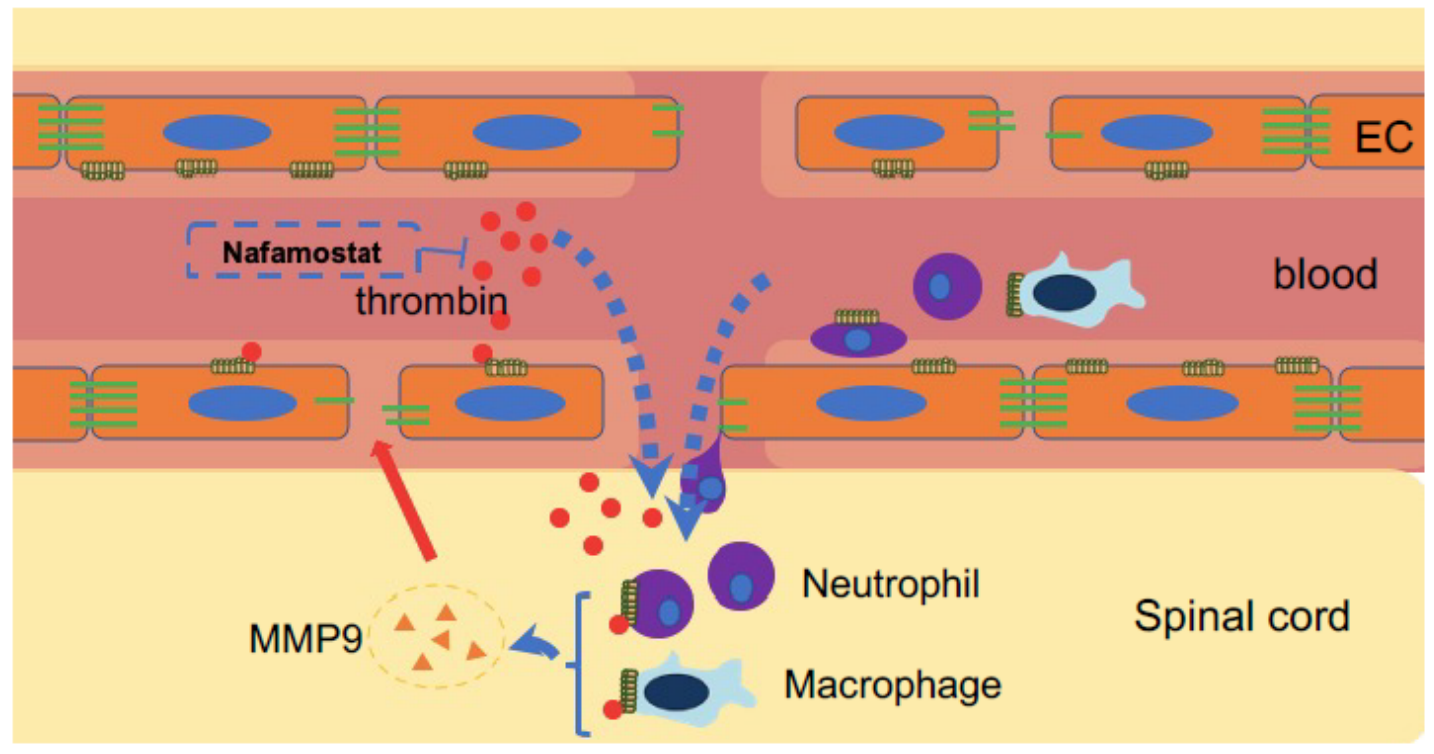

PAR1
TJPs
Endothelial cells
Nacrophage
Neutrophil
Vascular matrix
Spinal cord tissue

Figure 7

The repair Mechanism of Nafamostat Mesylate on spinal cord injury. Graphical abstract of mechanism of $\mathrm{NM}$ repairing $\mathrm{SCl}$

\section{Supplementary Files}

This is a list of supplementary files associated with this preprint. Click to download.

- Sfigure1.pdf

- Sfigure2.pdf

- Sfigure3.pdf

- Sfigure4.pdf

- Sfigure5.pdf

- Sfigure6.pdf 